\title{
BACTERIAL FIBRINOLYSIN, ITS POSSIBLE THERAPEUTIC APPLICATION IN TUBERCULOUS MENINGITIS
}

\author{
BY \\ I. A. B. CATHIE \\ From the Hospital for Sick Children, Great Ormond Street, London
}

(RECEIVED FOR PUBliCATION, NOVEMBER 12, 1948)

\begin{abstract}
A constant feature at autopsy in cases dying from tuberculous meningitis, whether treated with streptomycin or not, is a gelatinous exudate at the base of the brain. Frequently this is found in association with, or causing, hydrocephalus, and once the exudate has been formed it is difficult to visualize how streptomycin treatment alone could achieve its resolution. Even were streptomycin able to penetrate and sterilize the exudate the most likely end result would be its organization to fibrous tissue with an increase in the conditions leading to hydrocephalus.
\end{abstract}

The known propensity of cerebrospinal fluid from cases of tuberculous meningitis to form spider's web clots on standing which give the reactions of fibrin, combined with the fibrinous nature of the exudate, has led to the consideration of other forms of therapy in combination with streptomycin which might inhibit fibrin formation in the thecal space. Of the possible anticoagulants, heparin has received the most attention. The drawback that it forms a deposit with streptomycin may be avoided by spaced dosage or by the method of St. Hill and others (1948), in which heparin is added to streptomycin until no further deposition occurs, after which the supernatant fluid is removed by decantation. This supernatant fluid contains all the streptomycin used, and heparin may be added to it without the formation of a deposit.

Our experience of this combination has been disappointing as judged by the results seen in several patients at autopsy, and, indeed, although it is likely that heparin may prevent the deposition of further fibrin it is improbable that it would have any effect upon the fibrinous exudate, which presumably is already present when the diagnosis of tuberculous meningitis is made. For this reason some other means was sought which would have an action more of a lytic than of a preventive nature, and eventually it was decided to investigate the possibilities of the fibrinolysin produced by some strains of bacteria.

The fibrinolysin produced by pathogenic strains of haemolytic streptococci has been examined by Tillett and Garner (1933), Lancefield and Hare (1935), Colebrook and others (1935), and by others, and a few preliminary experiments with Group A haemolytic streptococci showed that when grown in broth they produced varying amounts of a substance which was capable of lysing human fibrin clots. The variability of yield of lysin from different strains of streptococcus, and even of day-today cultures from the same strain, was overcome when Dr. C. Lack drew my attention to the strain of Strep. pyogenes known as H.64, a strain known for its abundant production of fibrinolysin. Since then only this organism has been used, and it has given consistently good yields of lysin.

\section{Preparation of Fibrinolysin}

The method of preparation of the lysin has been essentially that described by Garner and Tillett (1934), with slight modifications, and consists of adsorbing fibrinolysin from a broth culture on to aluminium hydroxide, from which it is eluted with a phosphate buffer solution.

Preparation of alumina $-50 \mathrm{~g}$. of aluminium sulphate $\left(\mathrm{Al}_{2} \mathrm{SO}_{4}\right)_{3} .18 \mathrm{H}_{2} \mathrm{O}$ (Willstätter and Kraut, 1924) in $150 \mathrm{ml}$. of distilled water are heated at $55^{\circ} \mathrm{C}$. until dissolved. $500 \mathrm{ml}$. of a 15 per cent solution of ammonia are warmed to $55^{\circ}$, mixed with the alumina, and stirred vigorously for 30 minutes at 55 to $60^{\circ}$. The resulting precipitate is washed repeatedly with distilled water followed by decantation until no further smell of ammonia remains. Usually three or four washes are necessary. It is then washed with $100 \mathrm{ml}$. of the ammonia solution to decompose any traces of 
basic sulphate. The precipitate is finally washed two or three times with distilled water and either centrifuged or filtered through a Buchner funnel using No. 1 Whatman filter paper and negative pressure. Filtration is preferred to centrifuging as the resultant paste is drier. The alumina is now ready for use.

Broth culture.-Strep. pyogenes $\mathbf{H} .64$ is heavily seeded into $500 \mathrm{ml}$. of heart digest broth containing 2 per cent $\mathrm{NaCl}$ and 0.05 per cent glucose and incubated for eighteen hours. Much longer incubation does not give appreciably greater yields of fibrinolysin. After passage through a clarifying filter the broth is ready for adsorption.

Adsorption and elution of fibrinolysin. The alumina paste is added to approximately $500 \mathrm{ml}$. of the broth culture and the two are agitated together while the temperature is raised to $37^{\circ} \mathrm{C}$. in a waterbath. The mixture is maintained at this temperature for one hour with frequent stirring. It is then filtered through a Buchner funnel, and the deposit, on which the fibrinolysin is adsorbed, is washed by resuspension twice each in $250 \mathrm{ml}$. volumes of saline and distilled water, removing the fluid after each washing by filtration. After the last washing the paste should be as dry as possible.

The deposit is now resuspended in $100 \mathrm{ml}$. of $\mathrm{M} / 10$ phosphate buffer solution $\left(35.8 \mathrm{~g}\right.$. $\mathrm{Na}_{2} \mathrm{HPO}_{4}$ in $950 \mathrm{ml}$. of distilled water, adjusted to $\mathrm{pH} 7.3$ with concentrated $\mathrm{HCl}$, and made up to $1,000 \mathrm{ml}$.), thoroughly stirred, and then incubated at $37^{\circ} \mathrm{C}$. for thirty minutes. The alumina is then removed by filtration, and the fibrinolysin-containing filtrate is finally sterilized by means of a bacterial filter.

Although the first elution contains more lysin than do subsequent ones, second and third elutions are only slightly less potent and are well worth preparing. Later elutions are much weaker than the first few. All manipulations of the alumina are facilitated with an ordinary household egg-whisk.

\section{Properties of Fibrinolysin}

The solution prepared by the above technique consists of a somewhat variable amount of fibrinolysin in phosphate buffer. Attempts to remove the phosphate by dialysis have been unsuccessful. When dialysed with a cellophane membrane against frequent changes of distilled water there was still phosphate present after two weeks, and there was some loss of potency of the lysin. Against running tap water a deposit formed in the solution which, as the process was accompanied by total loss of lytic activity, presumably contained the fibrinolysin.

Assay of fibrinolysin.-Originally clots were prepared by the addition of an excess of calcium to plasma obtained from routine blood sedimentation rate samples of blood. In the stronger concentrations of lysin, however, the phosphate combined with the excess calcium, producing an opalescence through which the behaviour of $\overline{\bar{a}}$ clot was difficult to watch. Further, it was found that plasma obtained from different patients ga results not invariably repeatable. As an outcon of these observations the following method assay has been temporarily adopted.

From the same donor at the same time each day $0.4 \mathrm{ml}$. of blood is taken directly into $0.1 \mathrm{ml}$. of 3.8 per cent sodium citrate in a B.S.R. pipette, and the plasma so obtained is used in a dilution of $1:$ in saline. To a row of tubes each containing $0.5 \mathrm{~m}$ : of diluted plasma are added one drop of thrombin solution (containing approximately 4 units of thrombin) and $0.5 \mathrm{ml}$. of serial dilutions of fibring lysin. In the higher strengths of lysin no clot may appear, while in the greater dilutions a clot is formed within about two minutes, which is lysed in a peried of time which lengthens as the lysin is more diluter. Either the end-point may be taken as the dilution of fibrinolysin which inhibits clot formation, or the tubs may be incubated at $37^{\circ} \mathrm{C}$. for one hour, the end point being in that tube which has completely lysed the clat in this period. It is essential to include a control cipt with saline, as occasionally the naturally occurrifing plasma fibrinolysin (Macfarlane and Pilling, 1946) wïl lyse the clot formed in it.

Not all preparations of lysin prevent visibe clot formation, although the first elution frofin aluminium hydroxide is more likely to have thi property than the later ones. For this reason the dilution capable of lysing a standard clot in oge hour gives a more precise figure for comparison of potency with other batches of fibrinolysin.

Typical figures for such a titration of lysin are:

\begin{tabular}{|c|c|c|c|c|c|c|c|c|}
\hline $\begin{array}{c}\text { Dilution } \\
\text { of lysin }\end{array}$ & & 4 & 8 & 12 & 16 & 20 & 24 & 宁 \\
\hline \multirow{2}{*}{$\begin{array}{l}\text { Time for } \\
\text { clot to lyse } \\
\text { in minutes }\end{array}$} & $\begin{array}{c}\text { Batch } \\
\text { A }\end{array}$ & 3 & 7 & 19 & 45 & 1 & & 윽 \\
\hline & B & $\mathrm{NC}$ & $\mathrm{NC}$ & 3 & 8 & 21 & 49 & 1 胥 \\
\hline
\end{tabular}

In these examples the end point was taken as dilution capable of dissolving its clot in one hour, and with batch $A$ it was 16 , while with $B$ it was 34 . Batch B was therefore arbitrarily assumed to 24/16 as potent as batch A. Adjustments Of $_{\mathrm{f}}$ potency may be made either by dilution with distilled water or by concentration by low tempepature distillation.

Preparations of fibrinolysin vary in poteficy within considerable limits, although obtained bुy exactly the same method, so that some form of 
comparable assay is essential. Of successive elutions from the same alumina the fifth elution is usually about one-tenth as strong as the first.

Method of action.-A preparation of fibrinolysin was taken which permitted the initial formation of a clot in all dilutions with the standard plasma and thrombin. The time for clots to form and disappear was carefully noted. Then plasma and lysin were incubated together for $\mathbf{3 0}$ minutes, after which thrombin was added and the appearance and disappearance of clots were timed. Similarly thrombin and lysin were incubated, plasma was added, and clot behaviour was timed. In both these experiments the times for appearance and disappearance of the clots were the same as when plasma, thrombin, and lysin were added simultaneously. They were taken to indicate that lysin has no effect upon either thrombin or fibrinogen, and that when fibrinolysin is present in sufficient strength to prevent visible clot formation it is able to dissolve the clot as it is formed.

By varying the amounts of plasma or lysin in methods of assay, the action of the lysin can be shown to be quantitative.

Specificity of fibrinolysin.-It has been said (Tillett and Garner, 1933) that many strains of Strep. pyogenes produce a fibrinolysin specific for human fibrin. The fibrinolysin under discussion, however, will dissolve fibrin clots prepared from rabbit, mouse, and guinea-pig plasma, although the period is slightly longer than with human fibrin. The only failure has been with plasma from a guinea-pig with a heavy tuberculous infection.

Stability of fibrinolysin. - The remarkable stability of fibrinolysin has been emphasized by Garner and Tillett (1934). As judged by the time taken to lyse a standard clot there is no loss of potency after incubation at $37^{\circ} \mathrm{C}$. for 14 days. Similarly, after heating for 45 minutes at $95^{\circ} \mathrm{C}$., followed by storage in the cold, at room temperature, or at $37^{\circ} \mathrm{C}$., there is no loss of activity in 14 days. Activity is completely destroyed by autoclaving for one hour at $15 \mathrm{lb}$. pressure and by acid hydrolysis.

\section{Therapeutic Application}

Several pieces of fibrinous exudate were taken from the base of the brain of cases dying of tuberculous and coliform meningitis and subjected to the action of undiluted fibrinolysin. In each case lysis of the exudate occurred in from two to seven days, depending upon the amount of exudate used. In the tuberculous cases a very fine mesh of fibrous tissue was left. As controls pieces of brain and normal meninges were used, and these remained unaltered after incubation for a month.

Clots formed on standing in the cerebrospinal fluid from many cases of tuberculous meningitis were incubated with fibrinolysin with disappointing results. In a few cases lysis took place, but in many no effect was seen, contrasting markedly with the lysis obtained with control clots prepared from plasma. In view of the good results with the post-mortem exudates, however, it was decided to investigate the clinical application of fibrinolysin.

$1 \mathrm{ml}$. of a trial batch of fibrinolysin was injected into each of six normal thecas, and gave rise to cell counts of 500-800 polymorphs and protein rises of $0-10 \mathrm{mg}$. after 24 hours. This effect is similar to the pleocytosis and protein rise caused by streptomycin in a normal theca. $1 \mathrm{ml}$. of phosphate buffer without fibrinolysin was given into a normal theca and no alteration was seen in the cerebrospinal fluid after 24 hours, indicating that the irritant effect was due to the fibrinolysin and not to the phosphate. Many injections have been made intrathecally in cases of tuberculous meningitis, and usually a small increase in the existing cell count has been seen after the first three or four injections, again similar to the effect of streptomycin. In a typical case the cells rose from 200 to 280 per c.mm. 24 hours after fibrinolysin had been injected. After fibrinolysin had been given for four days no further increase in the cell count was seen, the greatest count being 390 . Thereafter the cell count fell steadily, and the fibrinolysin appeared to have no adverse effect on it. The cell response was polymorph in type, and reversion to the original 95 per cent lymphocytosis was seen after two weeks.

Both the hydrochloride and calcium chloride complex of streptomycin produce a deposit with fibrinolysin in phosphate buffer, much as they do with heparin. But a mixture of the fibrinolysin solution and streptomycin sulphate remains clear, and only this salt has been used.

The variability of different preparations of fibrinolysin has been noted, and one particular yield, while not being outstandingly lytic, caused gross increases in the cerebrospinal fluid cell count of the two patients to whom it was given, one count rising from 300 to 2,000 per c.mm. in 24 hours. No sequelae to the reactions were seen, but the batch of fibrinolysin was immediately withdrawn and another substituted for it. No other batch has had this toxic effect.

As the result of trial and error the amount of fibrinolysin to be used therapeutically has been 
chosen as 100 times the amount capable of lysing the standard clot in one hour. Thus, when a preparation lyses clots in a dilution of $1: 200,0.5 \mathrm{ml}$. are given diluted with the dose of streptomycin and $5-10 \mathrm{ml}$. of normal saline. When the strength of the fibrinolysin is less, proportionately greater amounts are given. With regard to frequency of administration, some cases have been given fibrinolysin intrathecally every time the streptomycin was given, while other cases have received it daily for the first fortnight of treatment only. Fibrinolysin has been given by the lumbar route, intracysternally, and into the lateral ventricles without apparent ill-effect.

\section{Discussion}

With regard to the various ways of demonstrating potency, both absolute and relative, of different yields of fibrinolysin, the following quotation from Macfarlane (1948) seems apposite: "The real or imaginary components of a theoretical mechanism can be separated to the investigator's satisfaction with little difficulty and allowed to interact in endless permutations and combinations. Each experiment suggests another; always the intangible solution seems just within reach and the experimenter is led deeper and deeper into his own, often unjustified, interpretations of his findings." With this observation in mind, only the crudest method of assay is mentioned here, and the many experiments carried out with varying amounts of components have been omitted.

Twelve batches of $100 \mathrm{ml}$. of fibrinolysin have now been prepared, and while no two batches have been strictly comparable in strength there has been a gradual increase of potency over the series. Thus, in the first batch prepared activity was demonstrable only at a dilution of $1: 20$, batch 12 was active with the same clot at $1: 320$, while the activity of the remaining batches fell between these figures. The one toxic batch prepared was discarded with its toxicity unexplained; it was bacteriologically sterile, and with the same operators, material, and apparatus for every batch prepared no useful line of investigation suggested itself.

It is unlikely that fibrinolysin prepared by this method does not contain varying amounts of other streptococcal toxins. Some of these may be destroyed by heat without detriment to the fibrinolysin, but as the preparation seems to have so little deleterious effect on tuberculous meninges this step has not been thought necessary.

An interesting and disappointing observation has been the infrequency with which fibrinolysin has been able to lyse the fibrin clots formed on standing in the cerebrospinal fluid from tuber- culous meningitis, while control plasma clots are lysed. Such cerebrospinal fluid has been tested without success for evidence of antifibrinolytio activity. The observation, taken with the failure to lyse plasma clots from a tuberculous guinea-pi产 suggests that there may be some qualitative differo ence between normal fibrin and that in tubero culosis, although the meningeal exudate is fairly readily lysed.

Fibrinolysin has been used almost entirely for tuberculous meningitis, but its possibilities in othey conditions with undesirable fibrin deposition have not been overlooked. In plastic peritonitis and pneumococcal empyema, for example, it might have a therapeutic place, and such cases are being investigated as they are encountered. It is possible too, that more potent, or more specific, fibrinolysin may be obtained from organisms other than the streptococcus, but as yet only this organism has been used.

As with the treatment of tuberculous meningitis with streptomycin alone, a long period of observa tion will be necessary to evaluate the efficacy of fibrinolysin as an adjuvant in this condition. Several cases have been under treatment with of for varying periods of time as yet too short warrant any other conclusion than that it may be given intrathecally without danger, and that pre liminary results have been sufficiently encouraging to justify an extended trial of the preparation.

The term fibrinolysin is used here to denote the bacterial product in the presence of which lysis of a fibrin clot occurs. It is probable that this lysis depends upon a compound enzyme system and that the bacterial factor is an activator of profibrinolysin, or plasminogen (Lack, 1948).

\section{Summary}

The preparation and assay of fibrinolysin from haemolytic streptococci are described.

Investigation of some of its properties and mo of action are outlined.

The therapeutic possibilities of fibrinolysin, pan ticularly with regard to tuberculous meningitis, are briefly indicated.

I wish to thank Mr. G. W. Cecil for much pains taking technical assistance.

REFERENCES

Colebrook, L., Maxted, W. R., and Johns, A. M. (1935). J. Path Bact., 41, 521.

Garner, R. L., and Tillett, W. S. (1934). J. exp. Med., 60, 239.

Lack, C. H. (1948). Nature, 101, 559.

Lancefield, R. C., and Hare, R.'(1935). J. exp. Med., 61, 335.

Macfarlane, R. G. (1948). J. clin. Path., 1, 113.

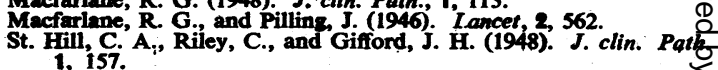

Tillett, W. S., and Garner, R. L. (1933): J. exp. Med., E3, 485.

Willstătter, R., and Kraut, H.(1924). Ber.disch. chem. Ges., 57,108 